Revista de Investigación de Física 22(2), (2019)

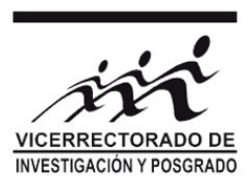

\title{
Vigilancia de la salinidad del suelo en cultivos de arroz en Lambayeque, periodo 2001-2014 utilizando imágenes de satélite y conductividad eléctrica
}

\author{
Rodrigo Sigueñas ${ }^{* 1}$, Leonidas Ocola ${ }^{1}$ y Jhon Guerrero ${ }^{1}$ \\ ${ }^{1}$ Universidad Nacional Mayor de San Marcos, Facultad de Ciencias Físicas, Lima, Perú
}

Recibido 05 noviembre 2019 - Aceptado 23 diciembre 2019

\section{Resumen}

El objetivo de este trabajo es estimar la salinidad del suelo en áreas de cultivos de arroz del distrito de Lambayeque e investigar su comportamiento espacio-temporal, utilizando imágenes de satélite de los sensores ETM+ de Landsat 7, MODIS/Terra y datos de conductividad eléctrica del suelo obtenido in situ. Los parámetros que se analizaron son el Índice de Vegetación de Diferencia Normalizada (NDVI), el Índice de Salinidad (IndSal) y la anomalía estandarizada de NDVI. El análisis del máximo valor compuesto (MVC) de NDVI e IndSal, la desviación estándar del NDVI e IndSal de las imágenes ETM+ y las anomalías estandarizada de los sensores ETM+ y MODIS permitieron separar los suelos con buena calidad agrícola de aquellos con problemas de salinidad. A partir del MVC de NDVI e IndSal se obtuvo 12864.24 ha y 12567.96 ha respectivamente pertenecientes a áreas de cultivos de arroz, la desviación estándar del NDVI e IndSal dio como resultado 12692.16 ha y 12250.53 ha de cultivos de arroz respectivamente, el área detectada a través de la conductividad eléctrica (CE) del suelo correspondiente a las clases "no salino" y "ligeramente salino" es de 12661.02 ha. De cada una de las áreas obtenidas se obtuvo un shapefile, posteriormente se obtuvo la variación temporal del NDVI del sensor MODIS, del cual se obtuvo el parámetro estacional SI y su correlación ( $R$ ) con los datos (superficie cosechada, producción y rendimiento) de cultivos de arroz proporcionados el Ministerio de Agricultura y Riego (MINAGRI) fueron muy significativos (0.74 - 0.90), esto indica que las áreas obtenidas si pertenecen a cultivos de arroz. Detectadas las áreas de cultivos de arroz se obtuvo la variación temporal de la conductividad eléctrica del suelo en el periodo 2001 - 2014. Palabras clave: ETM+, MODIS, NDVI, IndSal, IDL, Toolkit, TimeStats..

Monitoring of soil salinity in rice crops in Lambayeque, 2001-2014 period using satellite images and electrical conductivity

\section{Abstract}

The objective of this research work is to esrtimate soil salinity in rice crop areas in Lambayeque district and investigate the temporal and spatial behavior, using ETM+ sensor (LandSat-7) satellite images, MODIS sensor (TERRA) and data obtained from electrical conductivity (EC) of the soil in situ. For this purpose, normalized difference vegetation index (NDVI), soil salinity index (IndSal) and the standardized anomaly of the NDVI were the analysed parameters. The maximun compound value (MVC) analysis of the NDVI and soil salinity index, the standard deviation of the NDVI and soil salinity index from ETM+ sensor and standardized anomalies from ETM+ and MODIS sensors allowed to separate high quality soils from those with salinity problems. Based on the MVC de NDVI and soil salinity index it was obtained 12864.24 ha and 12567.96 ha respectively belonging to rice crop areas, the standard deviation of NDVI and IndSal resulted in 12692.16 ha and 12250.53 ha of rice crop areas respectively, the detected area by means of electrical conductivity of the soil, belonging to "non saline" and "slightly saline" is of 12661.02 ha. A shapefile was achieved from each analysed area, layer the temporary variation of NDVI was obtained from MODIS sensor, through which it was reached the seasonal index (SI) and its correlation (R) with the rice crop data (harvested area, producción and crop yields) provided by the Ministry of Agriculture and Irrigation (MINAGRI) were particularly significant $(0.74-0.90)$, this shows that the analysed areas belong to rice crops. Once the rice crop areas were detected, the temporary variation of the electrical conductivity of the soil between 2001-2014 was obtained.

Keywords: ETM+, MODIS, NDVI, IndSal, IDL, Toolkit, TimeStats..

\footnotetext{
${ }^{*}$ rodrigo.siguenas@unmsm.edu.pe
} 


\section{Introducción}

La salinización de los suelos es uno de los peligros ambientales que degrada las tierras de cultivo y su rehabilitación es muy costosa. Constituye uno de los principales problemas globales que afecta las bases económicas y sociales de muchos países en el mundo. En el Perú, la degradación de tierras en la costa se debe principalmente a la salinidad del suelo por la proximidad al océano. Aproximadamente, el $50 \%$ del área total cultivada se encuentra afectada en diferente grado por sales, siendo la más afectada la costa norte: Regiones de Lambayeque, Piura y La Libertad. Estas regiones son áreas importantes para la producción agrícola de exportación (Rojas y Campos, 2011; Badaracco, 2012; Soca, 2015) [Roj11] [Bad12] [Soc15].

Wiegand et al., (1996), realizaron estudios en parcelas de 15, 36, 59 hectáreas cultivadas con algodón (Gossipium hirsutum) y caña de azúcar (Saccharum officinarum) en California y Texas (Estados Unidos) y obtuvieron datos de salinidad y parámetros fisiológicos en sitios de muestreo, para calibrar imágenes de satélite Spot-1 HRV (Wiegand et al., 1996) [Wie96]. Las parcelas se clasificaron espectralmente y se desarrollaron modelos estadísticos a partir de los datos de campo y la información espectral, lo que condujo a estimar la salinidad de los pixeles de las parcelas estudiadas y a la obtención de mapas de clasificación por salinidad. La información espectral de las imágenes del satélite Spot-1 la obtuvieron de las bandas en las regiones espectrales verde, rojo e infrarrojo cercano. En el 2002, Quan et al., utilizaron la reflectancia multiespectral de imágenes de satélite y datos de conductividad eléctrica del suelo y NDVI, para verificar el uso de la teledetección como una herramienta de apoyo para el manejo de los cultivos, y desarrollaron una metodología para generar mapas de salinidad de suelos, que es el factor causante en el crecimiento de la caña de azúcar (Quan et al., 2003) Qua03. Zhang et al., estudiaron la relación existente entre los datos de NDVI y EVI en series de tiempo con salinidad de los suelos. Los resultados mostraron que la calidad de los datos de series de tiempo de los índices de vegetación (NDVI y EVI), mejorados con el filtro de Savitzky-Golay, proporcionan umbrales más precisos de etapas fenológicas de los cultivos. Los perfiles fenológicos de series de tiempo EVI suavizadas, presentaron una mejor relación "R" frente a los valores de CE (conductividad eléctrica) del suelo (0.65-0.95) siendo la CE del suelo el indicador del grado de salinidad (Zhang et al., 2015) [Zha15].

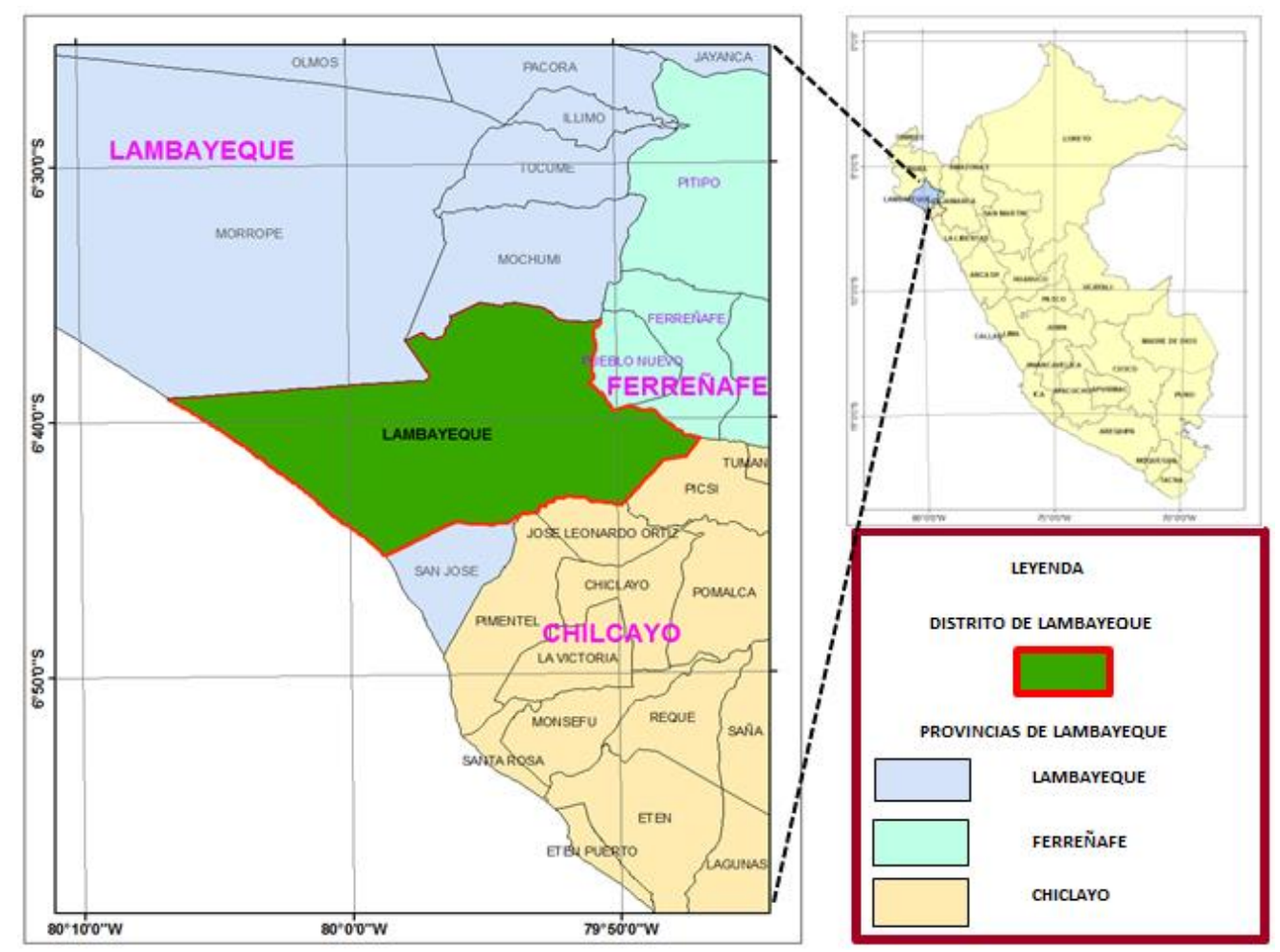

Figura 1: Ubicación del área de estudio, en color verde, el marco rojo representa el límite externo del distrito de Lambayeque. Fuente: Elaboración propia 
Desde el punto de vista agrícola, los suelos salinos son los que contienen suficientes sales solubles en la zona de raíz que afecta negativamente el crecimiento de las plantas, al hacer el agua del sub-suelo menos disponible para su extracción por las raíces, debido a la reducción del potencial osmótico del agua del suelo (Allen et al., 2006) [All06]. Las sales poseen afinidad por el agua, lo que se traduce en la necesidad del cultivo de gastar más energía metabólica y emplear una mayor fuerza mecánica para la extracción del agua de un suelo salino (Richards, 1954) [Ric54].

La CE se define como la conductividad eléctrica de la solución del agua del suelo después de añadir una cantidad de agua destilada suficiente para llevar el contenido de agua del suelo a punto de saturación (Rhoades et al., 1999) [Rho99]. La CE se expresa en decisiemens por metro $(\mathrm{dS} / \mathrm{m})$. Para efectos de la definición, los suelos que poseen una conductividad eléctrica mayor a $4 \mathrm{dS} / \mathrm{m}$ a $25^{\circ} \mathrm{C}$ se consideran suelos salinos Tabla 1 (Richards, 1954) [Ric54], Los cultivos de arroz son muy sensibles a la salinidad del suelo, el valor umbral de CE de los cultivos de arroz es 1.9 $\mathrm{dS} / \mathrm{m}$ Tabla 2 (Grattan et al., 2002) [Gra02].

\section{Datos y Metodología}

El área de estudio se encuentra en el distrito de Lambayeque, ubicado en la cuenca del río Chancay, provincia de Lambayeque, región Lambayeque (Núñez et al., 2006) Nuñ06. Entre las coordenadas 642'80"S, $79^{\circ} 54^{\prime} 56.77^{\prime \prime} \mathrm{W}$, con una extensión de 32827.68 ha aproximadamente (Figura 1).

\section{Datos}

Se obtuvo datos de "área cosechada, producción y rendimiento"de cultivo de arroz en el periodo 2001-2014 por cortesía del Ministerio de Agricultura y Riego MINAGRI. Los datos de conductividad eléctrica del suelo fueron proporcionados por el Laboratorio de Suelos de la Facultad de Agronomía de la Universidad Nacional Pedro Ruíz Gallo (UNPRG) de Lambayeque: 240 muestras en total.

La extracción de muestras de campo corresponde a una columna extraída hasta una profundidad de $0.30 \mathrm{~m}$, en puntos estratégicos del área de estudio, dentro de las parcelas de arroz. Los puntos de muestreo fueron debidamente georreferenciados con un GPS en el sistema WGS 84. Se obtuvo una muestra con un peso de 2 kilogramos aproximadamente, la conductividad eléctrica se determinó del extracto del suelo de cada muestra, mediante la técnica 1:1 en unidades del sistema Giorgi (dS/m). La extracción de las muestras de suelo se dio el 18 de Setiembre del 2007 y el 13 de Setiembre del 2007. En el momento de extracción de las muestras de suelo, las áreas de cultivos de arroz se encuentran en preparación para la instalación de cultivos de arroz. Las imágenes del sensor ETM+ de Landsat 7 son de acceso libre, (https://earthexplorer.usgs.gov). El porcentaje de nubosidad en el momento de la adquisición es importante para este estudio. Durante el periodo 20012014 se obtuvo un total de 71 imágenes nivel L1T con baja nubosidad.

Los productos de NDVI MODIS/Terra (MOD13Q1) son de acceso libre (https://1pdaacsvc.cr.usgs.gov/ appeears/). Se obtuvo 322 imágenes de NDVI generadas cada 16 días y $250 \times 250 \mathrm{~m}^{2}$ de resolución espacial.

\section{Metodología}

\section{Datos de campo}

Se realizó un control de calidad a los datos del MINAGRI, al igual que los datos de CE del suelo medidos en el laboratorio. Se verificó que cada uno de los datos pertenezca a áreas de cultivos de arroz.

\section{Datos de satélite}

Los datos imágenes de satélite, fueron sometidos a un pre-procesamiento y correcciones geométricas, posteriormente se determinó la radiancia espectral y la reflectancia espectral del suelo (Chander et al., 2009) [Cha09.

Se utilizó la imagen multiespectral de fecha 20/11/2007 para relacionar la reflectancia espectral del suelo con la CE del suelo medida en el laboratorio, para este fin se tomaron nueve pixeles teniendo como pixel central el punto de la muestra de CE en el suelo, de esta manera se obtuvo el promedio de la reflectancia espectral del suelo de nueve pixeles, posteriormente se analizó mediante regresión lineal simple y múltiple con la CE del suelo. Mediante la aplicación del método de regresión lineal, se obtuvo una ecuación con un coeficiente de Pearson de $R=0.85$.

El procesamiento de las imágenes de NDVI MODIS, se realizó con el software de procesamiento de imágenes ENVI y el lenguaje de programación IDL, mediante el módulo "MODIS Conversion Toolkit" (MCTK) desarrollado por White (2007) |Whi07]. Luego se aplicó el módulo TimeStats para corregir valores perdidos y errores producidos durante la toma de la imagen propuesta por Udelhoven(2010) [Ude10]. Posteriormente con los shapefiles de cada una de las clases de CE del suelo, se obtuvo las series temporales de los valores promedio de NDVI.

\section{Índice de Vegetación Diferencial Normalizado}

El Índice de Vegetación Diferencial Normalizado (NDVI) se obtuvo a partir de la reflectancia espectral del suelo del sensor ETM+ de Landsat 7. Este parámetro físico mide el vigor de las plantas, y está directamente relacionado con la capacidad fotosintética y a la absorción de energía. Se obtiene de la relación entre las longitudes de onda roja y casi-infrarroja, la clorofila absorbe gran parte del espectro visible (Rojo) y las estructuras de las plantas (Epidermis) por el gran contenido de moléculas de agua 
que estas presentan, reflejan fuertemente las ondas casiinfrarrojas (Huete et al., 2002; Lee, 2012; Gates et al., 1965) [Hue02] [Lee12] Gat65].

EI NDVI no mide directamente la productividad ni la disponibilidad de biomasa, pero si tiene una relación con el contenido de materia inorgánica del subsuelo donde se encuentra el cultivo, el daño que pueda originar la acumulación de sales (materia inorgánica) en el sub-suelo (zona de raíz del cultivo de arroz, $0.30 \mathrm{~m}$ de profundidad) se manifiesta directamente en el vigor de la planta y este afecta directamente el NDVI. Se obtuvo el NDVI de 71 imágenes multiespectrales del sensor ETM+ usando la ecuación 1.

$$
N D V I=\frac{\rho_{N I R}-\rho_{\text {red }}}{\rho_{N I R}+\rho_{\text {red }}}
$$

Se utilizó la reflectancia de la banda roja (entre 0.6 - $0.7 \mu \mathrm{m}$ ), y el infrarrojo cercano (entre $0.7-1.1 \mu \mathrm{m}$ ), correspondiente a las bandas 3 y 4 del sensor ETM+ respectivamente.

\section{Máximo valor compuesto de imágenes}

El máximo valor compuesto (MVC) consiste en seleccionar el mayor valor numérico de un pixel determinado en imá genes de diferentes fechas (Bai et al., 2005) [Bai05]. Se obtiene como resultado una imagen de valores máximos. Se generó el MVC de 71 imágenes de NDVI del sensor ETM+, periodo 2001 - 2014 para el área de estudio.

\section{Índice de salinidad}

El índice de salinidad (IndSal), es un parámetro utilizado en la detección de salinidad de suelos cultivables. Tiene una alta precisión para detectar la salinidad en la superficie del suelo desnudo, los valores fluctúan entre 1.0 y +1.0 , los valores próximos a -1.0 y 0.0 representan niveles altos de salinidad en la superficie del suelo, y los valores cercanos a +1.0 indican un nivel bajo de salinidad.

\begin{tabular}{lll}
\hline CE $(\mathbf{d S} / \mathbf{m})$ & Clase de la salinidad & Efectos \\
\hline $0-2$ & No salino & Suelos normales \\
$2-4$ & Ligeramente salino & Son afectados los rendimientos de los cultivos muy sensibles \\
$4-8$ & Moderadamente salino & Son afectados los rendimientos de la mayoría de los cultivos \\
$8-16$ & Fuertemente salino & Solo se obtienen rendimientos aceptables en los cultivos tolerantes \\
$>16$ & Extremadamente salino & Muy pocos cultivos dan rendimientos aceptables \\
\hline
\end{tabular}

Tabla 1: Clasificación de los suelos por rangos de CE (Richards, 1954) Ric54.

\begin{tabular}{|c|c|c|c|c|c|}
\hline \multirow{3}{*}{ Cultivo } & \multirow{3}{*}{$\begin{array}{l}\text { Valor umbral } \\
C E(d S / m)\end{array}$} & \multicolumn{4}{|c|}{ Pérdida de rendimiento } \\
\hline & & $10 \%$ & $25 \%$ & $50 \%$ & $100 \%$ \\
\hline & & $\mathrm{CE}(\mathrm{dS} / \mathrm{m})$ & $\mathrm{CE}(\mathrm{dS} / \mathrm{m})$ & $\mathrm{CE}(\mathrm{dS} / \mathrm{m})$ & $\mathrm{CE}(\mathrm{dS} / \mathrm{m})$ \\
\hline Arroz & 1.9 & 3.0 & 4.6 & 7.4 & 12.0 \\
\hline Algodón & 7.7 & 9.6 & 13.0 & 17.0 & 27.0 \\
\hline Caña de azúcar & 4.0 & 5.1 & 7.2 & 11.0 & 18.0 \\
\hline Trigo & 6.0 & 7.4 & 9.5 & 13.0 & 20.0 \\
\hline Frijoles & 1.0 & 1.5 & 2.3 & 3.6 & 6.5 \\
\hline
\end{tabular}

Tabla 2: Tolerancia de la salinidad en algunos cultivos (Grattan et al., 2002) [Gra02].

El índice de salinidad, es usado en suelos sin vegetación o suelos en barbecho (Al-Khaier, 2003) |Alk03]. En el cálculo del IndSal, los valores de reflectancia varían entre (1.55-1.75) y (2.08-2.35) $\mu \mathrm{m}$ respectivamente, para el sensor ETM+ corresponden a las bandas 5 y 7 respectivamente (ecuación 2).

$$
\text { IndSal }=\frac{\rho_{5}-\rho_{7}}{\rho_{5}+\rho_{7}}
$$

Se obtuvo 71 imágenes de IndSal en el periodo 2001 - 2014 del área de estudio.

\section{Desviación estándar de datos NDVI e IndSal}

La desviación estándar mide la variabilidad de un conjunto de datos con respecto al promedio (ecuación 3 ). Una desviación estándar alta indica que los datos se alejan significativamente del promedio, una desviación estándar baja indica que los datos son uniformes, cerca al valor promedio. En el área de estudio, los cultivos de arroz generan una alta variabilidad en el NDVI en todos los años. La desviación estándar del NDVI e IndSal de imágenes satelitales del sensor ETM+, ayudaron a localizar aquellas áreas de máxima variabilidad donde se instalan cultivos de arroz. 


$$
\sigma=\sqrt{\frac{1}{N-1} \sum_{i=1}^{N}\left(x_{i}-\bar{x}\right)^{2}}
$$

Donde: $\sigma$ : desviación estándar; $x_{i}$ : valor medido de NDVI; $\bar{x}$ : valor promedio de NDVI; $N-1$ : número de medidas.

\section{Serie Integral del NDVI}

Se obtuvo la serie integral (SI) de datos promedio de NDVI del sensor MODIS, pertenecientes a áreas de cultivos de arroz (suelos no salinos y ligeramente salinos). La SI, es un parámetro estacional, cuya definición es: "La integral de la diferencia entre la función que describe la estación y el nivel base de esta". Por lo tanto consiste en encontrar el área bajo la curva del NDVI durante el tiempo de productividad del cultivo de arroz (etapas: vegetativa, reproductiva y madurez), como se muestra en la Figura 2 (Fageria, 2013) [Fag13]. El significado ecológico es el siguiente: "Representa la suma o acumulación de la actividad fotosintética estacional, relacionado a la biomasa o producción primaria" (Zang et al., 2015; Kuenzer et al., 2015) [Zha15] [Kue15]. El resultado obtenido, se normaliza entre el periodo de producción para tener un resultado con valores de NDVI.

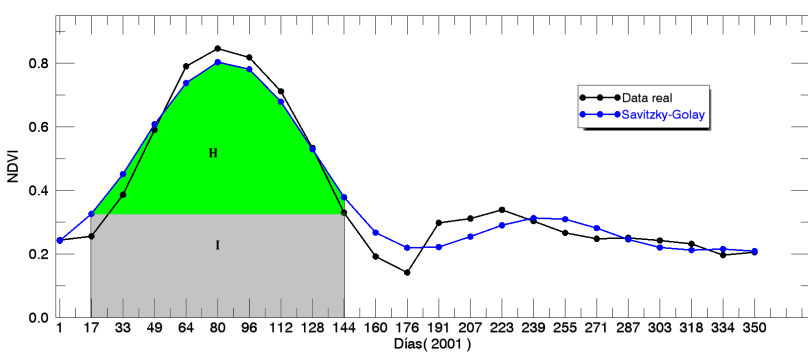

Figura 2: Variación anual del promedio de NDVI, datos obtenidos del máximo valor compuesto de NDVI. El parámetro estacional SI está representado por el color verde. Fuente: Elaboración propia

\section{Resultados y Discusión}

EI MVC del NDVI en un rango de $0.65-1.0$, detectó un total de 12864.24 ha que corresponden a áreas de cultivo en muy buen estado sin problemas de salinidad. A partir del MVC del IndSal en un rango de 0.35 - 1.0, se obtuvo 12567.96 ha de suelos de cultivo sin problemas de salinidad. Los resultados de la desviación estándar del NDVI y del IndSal dieron como resultado 12250.53 ha y 12692.16 ha respectivamente que corresponden a suelos de cultivos sin problemas de salinidad, o suelos donde se vienen instalando cultivos de arroz.

\begin{tabular}{|c|c|c|c|c|}
\hline Año & $\begin{array}{r}\text { SI } \\
(\mathrm{NDVI})\end{array}$ & $\begin{array}{l}\text { Área } \\
\text { cosechada } \\
{\left[(\text { ha }) \times 10^{4}\right]}\end{array}$ & $\begin{array}{l}\text { Producción } \\
{\left[(\mathrm{kg}) \times 10^{8}\right]}\end{array}$ & $\begin{array}{l}\text { Rendimiento } \\
{\left[(\mathrm{kg} / \mathrm{ha}) \times 10^{4}\right]}\end{array}$ \\
\hline 2001 & 0.37 & 1.1201 & 0.98663 & 0.8963 \\
\hline 2002 & 0.30 & 1.2894 & 1.02759 & 0.7970 \\
\hline 2003 & 0.29 & 1.2495 & 1.02810 & 0.8228 \\
\hline 2004 & 0.09 & 0.0497 & 0.02083 & 0.4191 \\
\hline 2005 & 0.29 & 0.6940 & 0.58097 & 0.8371 \\
\hline 2006 & 0.20 & 0.6354 & 0.48638 & 0.7655 \\
\hline 2007 & 0.23 & 0.5726 & 0.48673 & 0.8500 \\
\hline 2008 & 0.31 & 0.6313 & 0.54775 & 0.8677 \\
\hline 2009 & 0.35 & 0.9365 & 0.65536 & 0.6998 \\
\hline 2010 & 0.34 & 0.8585 & 0.66541 & 0.7751 \\
\hline 2011 & 0.23 & 0.5085 & 0.36104 & 0.7100 \\
\hline 2012 & 0.32 & 0.7260 & 0.60374 & 0.8316 \\
\hline 2013 & 0.34 & 0.8110 & 0.67154 & 0.8280 \\
\hline 2014 & 0.32 & 0.6230 & 0.51779 & 0.8311 \\
\hline
\end{tabular}

Tabla 3: Resultados del parámetro estacional SI en valores de NDVI, obtenidos con ayuda de un programa implementado en el lenguaje de programación IDL. Se muestran los datos de área cosechada, producción y rendimiento de los cultivos de arroz para el periodo 2001-2014, los valores en negrita, son anomalías producidas por eventos de sequía en la costa norte del Perú (Guerrero, 2015) [Gue15.

Se obtuvo los shapefiles que encierran en el rango de 0,65 - 1,0 del MVC del NDVI de las imágenes del sensor ETM+, y se aplicó a las imágenes de NDVI del sensor MODIS, obteniéndose la variación temporal del promedio espacial del NDVI periodo 2001-2014, de los cuales se obtuvo el parámetro fenológico de la SI de cada estación del cultivo de arroz, y se relacionó con la estadística del Ministerio de Agricultura Tabla 3 la relación ajustada a una función logarítmica, entre la SI de NDVI frente a los valores de área cosechada y producción, generó un coeficiente de relación $\mathrm{R}$ de 0.74 , y el $\mathrm{SI}$ frente al rendimiento se ajustó mediante una ecuación polinómica de tercer grado, generando un coeficiente de relación $\mathrm{R}$ de 0.90 mostrados en la Figura 3 .

A partir de los valores de CE del suelo y los valores de reflectancia espectral del suelo extraídas de la imagen multiespectral de fecha 20/11/2007 correspondientes a las bandas B1, B2, B3, B4, B5 y B7 del sensor ETM+. Se generó modelos de regresión lineal simple mediante el método de mínimos cuadrados Tabla 4 . Los modelos muestran la relación existente entre la reflectancia espectral del suelo y la CE del suelo, lo cual permite estimar la salinidad de los suelos en un espacio regional.

También se generó modelos de regresión lineal múltiple, utilizando el método de mínimos cuadrados, entre los valores de $C E$ del suelo y los valores de reflectancia espectral del suelo en las bandas B1, B2, B3, B4, B5 y B7 del sensor ETM+. Se obtuvieron modelos diferentes con respecto a los coeficientes de las bandas y a los inter- 
ceptos como se muestra en la Tabla 5 los coeficientes de correlación no presentaron mucha diferencia.

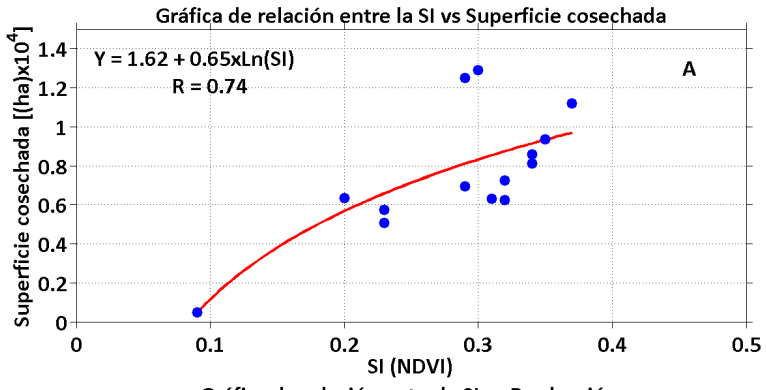

Gráfica de relación entre la SI vs Producción
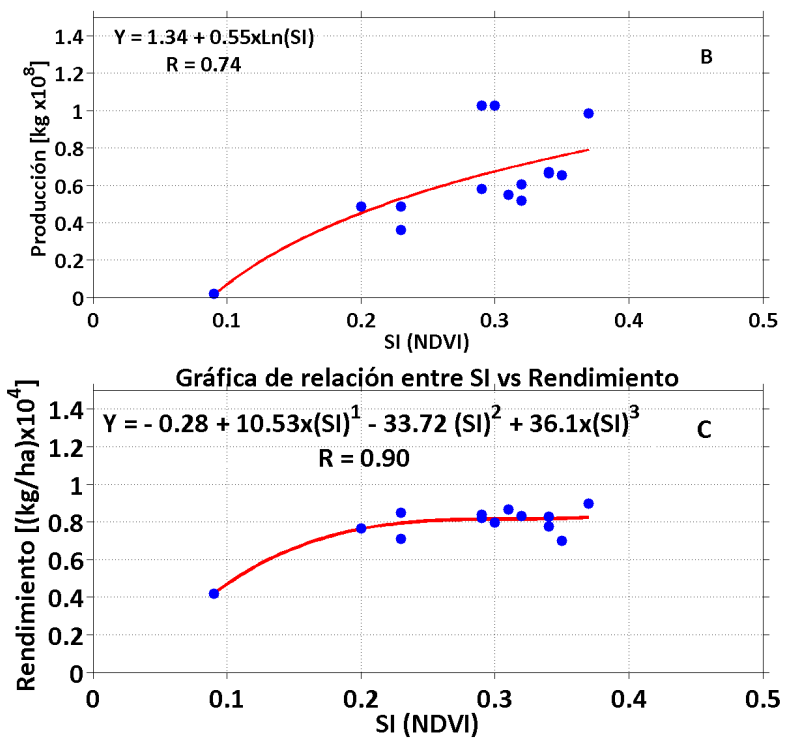

Figura 3: Las gráficas A, B y C muestran la relación entre la SI del NDVI del sensor MODIS y el área cosechada, producción y rendimiento de cultivos de arroz en el distrito de Lambayeque, en el periodo 2001 - 2014. Fuente: Elaboración propia

La ecuación 7 de la Tabla 5 se aplicó a la imagen multiespectral del sensor ETM+ de fecha 20/11/2007, obteniendo la distribución espacial de la CE del suelo, clasificándolo en cinco clases como se muestra en la Figura 4 según la Tabla 1 (Richards, 1954) [Ric54].

A partir de cada clase de la Figura 4 se obtuvo los shapefiles, para luego obtener la variación temporal del promedio espacial del NDVI del sensor MODIS de cada una de las clases obtenidas en la Figura 4 El área de los polígonos que encierran las clases de suelos no salinos y ligeramente salinos es de $12661.02 \mathrm{ha}$, pertenecientes a áreas de cultivos de arroz, por su alta variación del NDVI, como se muestra en la Figura 5, la línea de color magenta indica el umbral del NDVI de los cultivos de arroz (Ren et al., 2008) Ren08.

Como resultado final se obtuvo series temporales de anomalía estandarizada del NDVI del sensor MODIS, pa- ra el periodo 2001-2014, en la Figura 6 se muestra la variación temporal del promedio espacial de la anomalía estandariza del NDVI, en el año 2004 se observa una fuerte anomalía producto de un evento de sequía suscitado en la costa norte del Perú (Guerrero, 2015) Gue15].

A partir de la serie de anomalía estandarizada del NDVI del sensor MODIS, se escogió una imagen de fecha 16/10/2007 próxima a la extracción de muestras de suelos. Se obtuvo los valores de anomalía estandarizada correspondiente a los pixeles de las muestras de suelo, y evaluadas la relación mediante un modelo de regresión lineal simple, se generó una ecuación de la CE del suelo en función de la anomalía estandarizada del NDVI con un coeficiente de correlación " $R$ " negativo muy significativo de -0.73. Se aplicó el modelo obtenido a las imágenes de anomalía estandarizada de NDVI, y con ayuda de los vectores polígonos que encierran las áreas de las clases no salinas y ligeramente salinas de la Figura 4 se obtuvo la variación temporal de la CE del suelo en cultivos de arroz del distrito de Lambayeque (Figura 7).

\begin{tabular}{clll}
\hline EC. & Correlación & $\begin{array}{l}\text { Modelo } \\
\text { de regresión }\end{array}$ & $\mathbf{R}$ \\
\hline 1 & CE vs $\rho_{B 1}$ & $\mathrm{CE}=133.57 \times \mathrm{B} 1-8.60$ & $\mathrm{R}=0.835$ \\
2 & $\mathrm{CE}$ vs $\rho_{B 2}$ & $\mathrm{CE}=109.01 \times \mathrm{B} 2-8.47$ & $\mathrm{R}=0.823$ \\
3 & $\mathrm{CE}$ vs $\rho_{B 3}$ & $\mathrm{CE}=85.97 \times \mathrm{B} 3-7.33$ & $\mathrm{R}=0.830$ \\
4 & $\mathrm{CE}$ vs $\rho_{B 4}$ & $\mathrm{CE}=57.91 \times \mathrm{B} 4-6.31$ & $\mathrm{R}=0.639$ \\
5 & $\mathrm{CE}$ vs $\rho_{B 5}$ & $\mathrm{CE}=45.21 \times \mathrm{B} 5-5.30$ & $\mathrm{R}=0.762$ \\
6 & $\mathrm{CE}$ vs $\rho_{B 7}$ & $\mathrm{CE}=43.11 \times \mathrm{B} 7-3.36$ & $\mathrm{R}=0.793$ \\
\hline
\end{tabular}

Tabla 4: de regresión lineal simple entre la CE $(\mathrm{dS} / \mathrm{m})$ del suelo y los valores de reflectancia espectral del suelo de las bandas 1, 2,3, 4, 5 y 7 del sensor ETM+-LandSat-7(39 muestras de $\mathrm{CE}$ ).

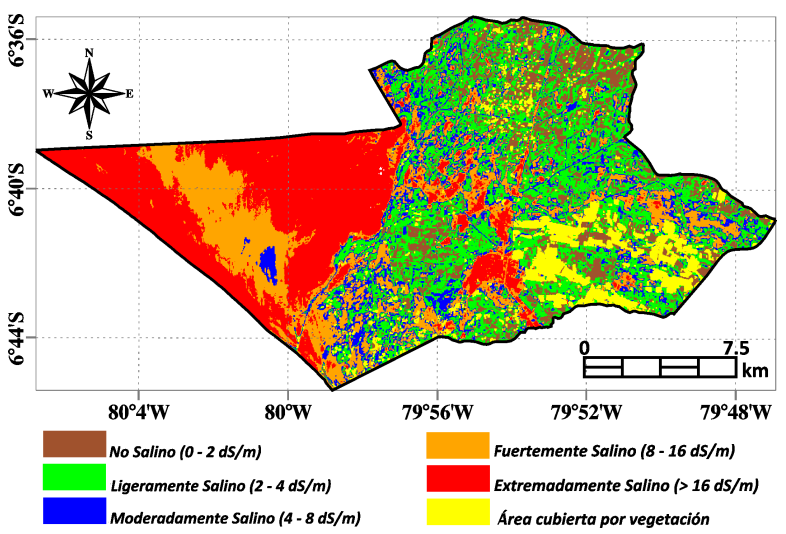

Figura 4: Mapa temático de la salinidad del suelo en el distrito de Lambayeque obtenido con el modelo de regresión lineal múltiple 7 de la Tabla 3 Las áreas de color amarillo pertenecen a otros cultivos como caña de azúcar y pastos. Fuente: Elaboración propia. 


\begin{tabular}{|c|c|c|c|}
\hline $\mathrm{N}^{\circ}$ & Ecuación & $R^{2}$ & $\mathrm{R}$ \\
\hline 1 & $C E=92.45 \times B 1+35.29 \times B 2-8.76$ & 0.7 & 0.84 \\
\hline 2 & $C E=76.30 \times B 1+38.29 \times B 3-8.29$ & 0.71 & 0.84 \\
\hline 3 & $C E=119.73 \times B 1+11.38 \times B 4-9.30$ & 0.71 & 0.84 \\
\hline 4 & $C E=107.20 \times B 1+11.54 \times B 5-8.48$ & 0.71 & 0.84 \\
\hline 5 & $C E=102.08 \times B 1+11.88 \times B 7-7.65$ & 0.71 & 0.84 \\
\hline 6 & $C E=12.84 \times B 2+75.33 \times B 3-7.49$ & 0.69 & 0.83 \\
\hline 7 & $C E=85.31 \times B 1-38.37 \times B 2+62.16 \times B 3-7.93$ & 0.72 & 0.85 \\
\hline 8 & $\mathrm{CE}=83.12 \times \mathrm{B} 1+28.96 \times \mathrm{B} 3+5.86 \times \mathrm{B} 4-8.73$ & 0.71 & 0.84 \\
\hline 9 & $C E=95.03 \times B 1-45.37 \times B 2+55.46 \times B 3+6.95 \times B 4$ & 0.71 & 0.84 \\
\hline 10 & $\mathrm{CE}=93.68 \times \mathrm{B} 1-52.47 \times \mathrm{B} 2+52.69 \times \mathrm{B} 3+4.78 \times \mathrm{B} 4+6.19 \times \mathrm{B} 7-7.74$ & 0.71 & 0.84 \\
\hline 11 & $\mathrm{CE}=87.50 \times \mathrm{B} 1-50.49 \times \mathrm{B} 2+55.82 \times \mathrm{B} 3+8.08 \times \mathrm{B} 7-7.28$ & 0.71 & 0.84 \\
\hline 12 & $\mathrm{CE}=97.07 \times \mathrm{B} 1-56.79 \times \mathrm{B} 2+53.87 \times \mathrm{B} 3+1.32 \times \mathrm{B} 4+7.17 \times \mathrm{B} 5+1.55 \times \mathrm{B} 7-7.79$ & 0.71 & 0.84 \\
\hline
\end{tabular}

Tabla 5: Modelos de regresión lineal múltiple para estimar un mapa de conductividad eléctrica del suelo $(\mathrm{dS} / \mathrm{m})$ a partir de datos de reflectancia del suelo de las bandas B1, B2, B3, B4, B5 y B7 sensor ETM+-Landsat-7 y datos de conductividad eléctrica del suelo (datos de campo).

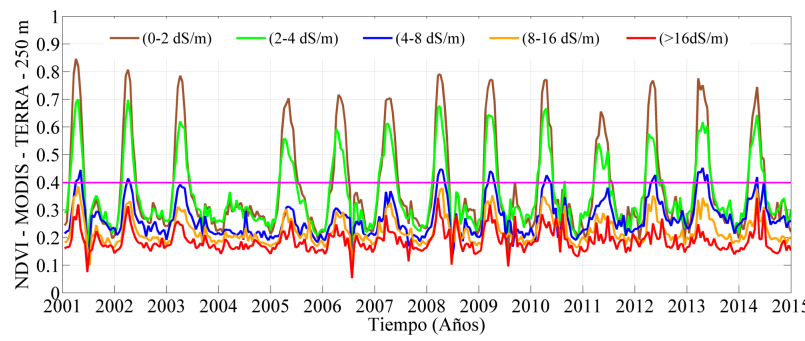

Figura 5: Serie temporal de datos promedio de NDVI del sensor MODIS para las clases de suelos mostrados en la Figura 4 Fuente: Elaboración propia.

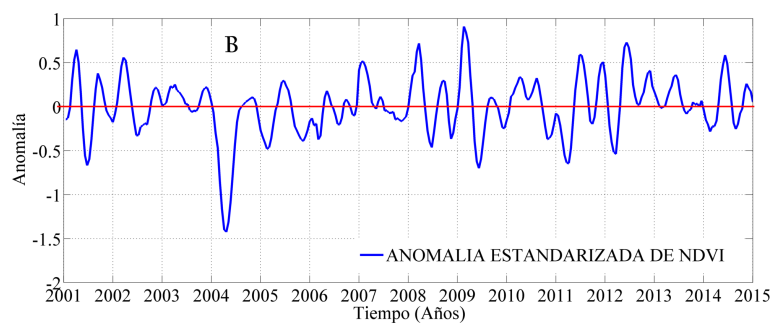

Figura 6: Variación temporal del promedio espacial de la anomalía estandarizada del NDVI para el periodo 2001 - 2014 del sensor MODIS. Fuente: Elaboración propia.

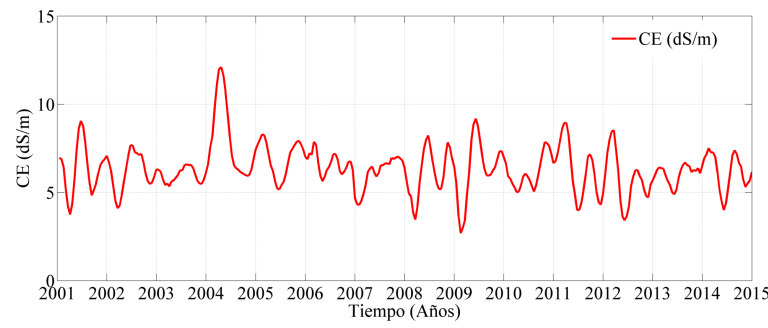

Figura 7: Variación temporal del promedio de la CE del suelo en áreas de cultivos de arroz del distrito de Lambayeque para el periodo 2001-2014 a una resolución temporal de 16 días.

\section{Conclusiones}

EI MVC del NDVI e IndSal son técnicas que ayudan a localizar las áreas de cultivos sin problemas de salinidad. En el distrito de Lambayeque a partir del MVC de NDVI e IndSal se obtuvo 12864.24 ha y 12567.96 ha respectivamente áreas de cultivo de arroz sin problemas de salinidad. La desviación estándar del NDVI e IndSal verificaron los resultados obtenidos con el MVC dando como resultados 12692.16 ha y 12250.53 ha respectivamente. Los vectores polígonos que encierran estas áreas, obtenidas del MVC y desviación estándar de imágenes del sensor $\mathrm{ETM}+$, presentan similares áreas, la diferencia más resaltante de las áreas obtenidas mediante la desviación estándar, es la discriminación con alta precisión de los caminos, canales de riego y drenajes situación que no sucede con el MVC. La variación temporal del promedio espacial del NDVI de imágenes MODIS, obtenidas con los vectores polígonos del MVC y desviación estándar, muestran similar variación debido a la resolución espacial de las imágenes del sensor MODIS. La alta relación existente entre el parámetro fenológico SI y los datos estadísticos del Ministerio de Agricultura, demostró que las áreas obtenidas con el MVC y desviación estándar, si pertenecen a cultivos de arroz.

El vector polígono que engloba las áreas de suelos no salino y ligeramente salino, presenta un total de 12661.02 ha y la variación temporal del promedio espacial de NDVI del sensor MODIS, confirma la instalación de cultivos de arroz. La variación temporal del promedio espacial de la CE del suelo, en áreas de cultivos de arroz (Figura 7), muestra una alta CE del suelo. Esto significa una pérdida aproximadamente del $10 \%$ de la producción del cultivo de arroz en el área de estudio, siendo el umbral de CE del suelo para este cultivo $1.9 \mathrm{dS} / \mathrm{m}$ según la Tabla 2 .

Además, la Figura 7 muestra un incremento de la CE 
del suelo cuando este se encuentra en reposo $\circ$ barbecho y disminuye cuando se instala el cultivo de arroz, esta disminución no es suficiente para un buen desarrollo del cultivo de arroz, aún sigue siendo perjudicial (Grattan et al., 2002) Gra02, frente a este problema se deben tomar las medidas correctivas, para controlar la degradación del suelo por la salinidad, a través de un eficiente sistema drenaje de aguas residuales o la utilización de yeso agrícola, para mejorar la producción de los cultivos de nuestros agricultores.

\section{Agradecimientos}

Los autores agradecen al Laboratorio de Teledetección (LABTEL) - Facultad de Ciencias Físicas - UNMSM, por la formación brindada. Así mismo, se agradece al Laboratorio de Suelos de la Facultad de Agronomía-UNPRG y MINAGRI, por las facilidades de campo para la obtención de los datos de CE del suelo y cultivos de arroz. Se agradece a la Administración Nacional de la Aeronáutica y del Espacio (NASA) por la obtención de las imágenes de los satélites LandSat y MODIS.

\section{Referencias}

[Alk03] Al-Khaier, F. (2003, March). Soil salinity detection using satellite remote sensing. ITC.

[All06] Allen, R., Pereira, L., Raes, D. y Smith, M. (2006). Evapotranspiración del cultivo: guías para la determinación de los requerimientos de agua de los cultivos. Roma: FAO, 298, 0.

[Bad12] Badaracco R. (2012). Detección de la salinidad en los suelos de cultivos de algodón usando imágenes de satélite en la costa norte del Perú Tesis de Licenciatura, Universidad Nacional Mayor de San Marcos, Lima, Perú.

[Bai05] Bai, Z. Bartholomeus, H., Schaepman, M. (2005). Assessing land degradation and improvement using NASA GIMMS, Shaanxi, China. In Remote sensing and geoinformation processing in the assessment and monitoring of land degradation and desertification (pp. 128-135).

[Cha 09] Chander, G., Markham, B. y Helder, D. (2009). Summary of current radiometric calibration coefficients for Landsat MSS, TM, ETM+, and EO-1 ALI sensors. Remote Sensing of Environment, 113(5), 893-903.

[Fag13] Fageria, N. (2013). Mineral nutrition of rice. CRC Press. New York.

[Gat65] Gates, D. Keegan, H. Schleter, J. y Weidner, V. (1965). Spectral properties of plants. Applied Optics, 4(1), 11-20.

[Gue15] Guerrero, J. (2015). Investigación de la climatología de la humedad atmosférica y su relación con la sequía en la costa peruana utilizando imágenes de satélite del sensor MODIS/TERRA. Tesis de Maestría, Universidad Nacional Mayor de San Marcos, LimaPerú.
[Gra02] Grattan, S., Zeng, L., Shannon, M. y Roberts, S. (2002). Rice is more sensitive to salinity than previously thought. California Agriculture, 56(6), 189198.

[Hue02] Huete, A., Didan, K., Miura, T., Rodriguez, E., Gao, X., Ferreira, L. (2002). Overview of the radiometric and biophysical performance of the MODIS vegetation indices. Remote Sensing of Environment, 83(1-2), 195-213.

[Kue15] Kuenzer, C., Dech, S. y Wagner, W. (2015). Remote sensing time series. Remote Sensing and Digital Image Processing, 22.

[Lee12] Lee, T. (Ed.). (2012). Irrigation Systems and Practices in Challenging Environments. BoD-Books on Demand.

[Nuñ06] Núñez, S., Rivera, R., Chira, J., Villacorta, S. (2006). Estudio geoambiental de la cuenca del río Chancay-Lambayeque. Informe Técnico, Biblioteca Central, Universidad Nacional Mayor de San Marcos, Lima-Perú.

[Qua03] Quan, A. Lhermitte, S., Gilliams, S. y Pérez, M. (2003). Relación de la salinidad del suelo con la reflectancia multiespectral de la caña de azúcar cultivada en condiciones extremas. Revista Ciencias Técnicas Agropecuarias, 12(3), 19-29.

[Ren08] Ren, J., Chen, Z., Zhou, Q. y Tang, H. (2008). Regional yield estimation for winter wheat with MODIS-NDVI data in Shandong, China. International Journal of Applied Earth Observation and Geoinformation, 10(4), 403-413.

[Ric54] Richards, L. (1954). Diagnosis and improvement of saline and alkali soils (Vol. 78, No. 2, p. 154). LWW.

[Rho99] Rhoades, J. Chanduvi, F. y Lesch, S. (1999). Soil salinity assessment: Methods and interpretation of 
electrical conductivity measurements (No. 57). Food y Agriculture Org.

[Roj11] Rojas, J. y Campos, E. (2011). Relación de la conductividad eléctrica de los suelos con la reflectancia de imágenes de satélite en la región de Lambayeque-Perú. Revista de Investigación de Física, 14(02).

[Soc15] Soca, R. (2015). Identificación de tierras degradadas por salinidad del suelo en los cultivos de caña de azúcar en Pomalca usando imágenes de satélite. Facultad de Ciencias Físicas-Universidad Nacional de San Marcos, 1-156.

[Ude10] Udelhoven, T. (2010). TimeStats: A software tool for the retrieval of temporal patterns from global satellite archives. IEEE Journal of Selected Topics in Applied Earth Observations and Remote Sensing, 4(2), 310-317.

[Whi07] White, D. (2007). The MODIS conversion toolkit (MCTK) user's guide. ITT Visual Information Solutions. Available online: http://nsidc.org/data/ modis/tools.html (accessed on 20 April 2014).

[Wie96] Wiegand, C., Anderson, G., Lingle, S. y Escobar, D. (1996). Soil salinity effects on crop growth and yield-Illustration of an analysis and mapping methodology for sugarcane. Journal of Plant Physiology, 148(3-4), 418-424.

[Zha15] Zhang, T. Qi, J. Gao, Y., Ouyang, Z. Zeng, S. y Zhao, B. (2015). Detecting soil salinity with MODIS time series VI data. Ecological Indicators, 52, 480489. 